\title{
Thermodynamical transcription of the density functional theory with constant temperature
}

\author{
Á. Nagy \\ Department of Theoretical Physics, University of Debrecen, H-4002 Debrecen, Hungary
}

(Dated: April 19, 2017)

\begin{abstract}
The thermodynamical interpretation of the density functional theory for an electronic ground state is revisited. Ghosh, Berkowitz and Parr invented the thermodynamical transcription of the ground-state density functional theory into a local thermodynamics. They introduced the idea of the local temperature that varies from point to point. The local temperature is defined via the kinetic energy density. The kinetic energy density is not uniquely defined, usually the everywhere positive gradient form is applied. Now we prove that it is possible selecting the kinetic energy density so that the local temperature be a constant for the whole system under consideration. The kinetic energy density is proportional to the electron density and the temperature is proportional to the kinetic energy. Further, the kinetic energy density corresponding to the constant temperature, maximizes the information entropy.
\end{abstract}




\section{INTRODUCTION}

More than three decades ago Ghosh, Berkowitz and Parr wrote a paper [1] entitled "Transcription of ground-state density functional theory into a local thermodynamics". They obtained a phase-space distribution function $f(\mathbf{r}, \mathbf{p})$ by maximizing a phase-space Shannon information entropy subject to the conditions that $f$ yields the density $n(\mathbf{r})$ and the local kinetic energy density of the system. A local Maxwell-Boltzmann distribution function was resulted. They introduced the concept of local temperature. This formalism has been used in several applications [3, 4]. For example approximate expressions for the exchange energy were derived $[2,5]$. It was possible to define local entropy, free energy and to establish an analogy with the classical thermodynamics of fluids [3]. There have been several extension of the formalism [6-16].

The local temperature of Ghosh, Berkowitz and Parr was defined via the kinetic energy density and varies from point to point. However, the kinetic energy density is not uniquely defined. Adding a term that integrates to zero to the kinetic energy density results another kinetic energy density with the same kinetic energy but different local temperature. Usually the gradient form of the kinetic energy density is applied, because it is everywhere positive.

Now we prove that it is possible selecting the kinetic energy density so that the local temperature be a constant for the whole system under consideration. The kinetic energy density is proportional to the electron density and the temperature is proportional to the kinetic energy. Moreover, the kinetic energy density corresponding to the constant temperature, maximizes the information entropy. This very simple interpretation gives a new insight into the density functional theory. Analytical examples for the linear harmonic oscillator and the H-atom are presented.

In the following section the Ghosh-Berkowitz-Parr theory is summarised. Section III. presents the new theory of the constant temperature. In Section IV the maximum entropy

principle is applied to derive the kinetic energy density. The last section is devoted to the examples of the linear harmonic oscillator and the H-atom. 


\section{A "THERMODYNAMICAL" TRANSCRIPTION OF DENSITY FUNC- TIONAL THEORY}

First the Ghosh-Berkowitz-Parr theory [1] is summarized. A system of $N$ electrons in a local external potential $v(\mathbf{r})$ is considered. The ground-state electron density $n(\mathbf{r})$ determines $v(\mathbf{r})$ and the ground-state total energy takes its minimum at the true density as HohenhergKohn theorems [17] say. As Kohn and Sham [18] showed there exists a non-interacting system, where the electrons move independently in a common, local (Kohn-Sham) potential.

Ghosh, Berkowitz and Parr defined a phase-space distribution function $f(\mathbf{r}, \mathbf{p})$ that satisfies

$$
\begin{aligned}
& \int d \mathbf{p} f(\mathbf{r}, \mathbf{p})=n(\mathbf{r}), \\
& \int d \mathbf{r} n(\mathbf{r})=N,
\end{aligned}
$$

and

$$
\int d \mathbf{p} \frac{p^{2}}{2 m} f(\mathbf{r}, \mathbf{p})=t_{s}(\mathbf{r})
$$

$m$ is the mass. The non-interacting kinetic energy density $t_{s}(\mathbf{r})$ integrates to the noninteracting kinetic energy $E_{k i n}$

$$
E_{k i n}=\int d \mathbf{r} t_{s}(\mathbf{r})
$$

There exist several distribution functions that satisfy the marginal conditions (1-3). The general forms of distribution functions satisfying somewhat different marginal conditions were studied by Cohen [19]. Ghosh, Berkowitz and Parr selected a distribution function by maximizing the entropy

$$
\begin{gathered}
S=\int d \mathbf{r} s(\mathbf{r}) \\
s(\mathbf{r})=-k \int d \mathbf{p} f(\ln f-1)
\end{gathered}
$$

subject to the constraints of correct density ( Eq. (1)) and correct non-interacting kinetic energy ( Eq. (3)). $k$ is the Boltzmann constant. The maximum entropy is obtained with a local Maxwell-Boltzmann distribution function

$$
f(\mathbf{r}, \mathbf{p})=e^{-\alpha(\mathbf{r})} e^{-\beta(\mathbf{r}) p^{2} / 2 m},
$$


where $\alpha(\mathbf{r})$ and $\beta(\mathbf{r})$ are $\mathbf{r}$-dependent Lagrange multipliers. The local temperature $T(\mathbf{r})$ is defined in terms of the non-interacting kinetic energy density

$$
t_{s}(\mathbf{r})=\frac{3}{2} n(\mathbf{r}) k T(\mathbf{r})
$$

i.e. by the ideal gas expression. Eqs. (3), (7) and (8) lead to

$$
\beta(\mathbf{r})=\frac{1}{k T(\mathbf{r})}
$$

and

$$
f(\mathbf{r}, \mathbf{p})=[2 \pi m k T(\mathbf{r})]^{-3 / 2} n(\mathbf{r}) e^{-p^{2} / 2 m k T(\mathbf{r})} .
$$

Therefore the distribution can be rewritten as

$$
f(\mathbf{r}, \mathbf{p})=e^{\mu / 2 k T(\mathbf{r})} e^{-\left(p^{2} / 2 m+v_{e f f}\right) / k T(\mathbf{r})},
$$

where

$$
v_{e f f}=\mu-\frac{\ln \lambda^{3} n}{\beta}
$$

and

$$
\lambda=\frac{1}{\sqrt{2 \pi m k T}} .
$$

The effective potential $v_{\text {eff }}$ is not the Kohn-Sham potential, but there is a simple relation hetween them [1].

\section{THE THEORY WITH CONSTANT TEMPERATURE}

According to Eq. (8), generally the local temperature varies from point to point. Moreover, it is not uniquely defined as the kinetic energy density is not unique. Adding a term that integrates to zero to the kinetic energy density results another kinetic energy density

with the same kinetic energy but different local temperature. Usually the gradient form of the kinetic energy density is applied, because it is everywhere positive.

Now, we prove that one can find a kinetic energy density so that the local temperature be a constant for the whole system under consideration. The kinetic energy density can be written as

$$
t_{s}(\mathbf{r})=\frac{3}{2} n(\mathbf{r}) k T(\mathbf{r})=t_{\text {grad }}+g(\mathbf{r})
$$


where

$$
t_{\text {grad }}(\mathbf{r})=\frac{\hbar^{2}}{2 m} \sum_{i=1}^{N}\left|\nabla^{2} u_{i}\right|^{2}
$$

is the well-known gradient form of the kinetic energy density and the function $g(\mathbf{r})$ integrates to zero:

$$
\int d \mathbf{r} g(\mathbf{r})=0
$$

$u_{i}$ are the orbitals. Certainly, $t_{\text {grad }}$ integrates to the non-interacting kinetic energy $E_{k i n}$

$$
E_{k i n}=\int d \mathbf{r} t_{\text {grad }}(\mathbf{r})
$$

From Eq. (14) we can obtain that

$$
g(\mathbf{r})=\frac{3}{2} \frac{n}{\beta}+t_{\text {grad }}
$$

For a constant $\beta$

$$
E_{k i n}=\int d \mathbf{r} \frac{3}{2} \frac{n}{\beta}=\frac{3}{2} \frac{N}{\beta} .
$$

Therefore

$$
\frac{1}{\beta}=k T=\frac{2}{3} E_{k i n} .
$$

Eqs. (17) and (20) lead to

$$
g(\mathbf{r})=t_{\text {grad }}+\frac{E_{k i n}}{N} n(\mathbf{r})
$$

and

$$
t_{s}(\mathbf{r})=\frac{E_{k i n}}{N} n(\mathbf{r}) .
$$

That is, the kinetic energy density is proportional to the electron density and the temperature is proportional to the kinetic energy.

Substituting Eq. (7) into Eq. (5) the well-known Sackur-Tetrode expression

$$
S=-k \int n(\mathbf{r}) \ln n(\mathbf{r}) d \mathbf{r}+\frac{1}{2} k \int n(\mathbf{r})[3 \ln (2 \pi m k T)+5] d \mathbf{r}
$$

is obtained for the entropy. $S$ can also be writen as

$$
S=-k \int n(\mathbf{r}) \ln n(\mathbf{r}) d \mathbf{r}+\frac{1}{2} k N\left[3 \ln \left(\frac{4 \pi m k E_{k i n}}{N}\right)+5\right] .
$$




\section{EXTREMUM INFORMATION ENTROPY PRINCIPLE}

In this section it is shown that the extremum entropy principle also leads to the constant temperature. Consider the Sackur-Tetrode expression (23) of the entropy. Find the function $g(r)$ that maximizes the entropy (23) and satisfies condition (16):

$$
k \int n(\mathbf{r})\left(-\ln n(\mathbf{r})+\frac{3}{2}\left[\ln (4 \pi m)-\ln (3 n)+\ln \left(t_{\text {grad }}+g\right)+\frac{5}{3}\right]\right) d \mathbf{r}-\zeta \int g(\mathbf{r}) d \mathbf{r}
$$

is maximum, where $\zeta$ is the Lagrange multiplier. Note that we search $g(r)$ with keeping the orbitals and the density fixed. The variation leads to

$$
\frac{3}{2} \frac{k n}{\left(t_{\text {grad }}+g\right.}-\zeta=0
$$

It can also be written as

$$
t_{s}=t_{\text {grad }}+g=\frac{3}{2} \frac{k n}{\zeta}
$$

Comparing with Eq. (14) we obtain

$$
\zeta=\frac{1}{k T}=\beta .
$$

That is, $\zeta$ equals the inverse temperature $\beta$. As the Lagrange multiplier $\zeta$ is a constant, the temperature $T$ should also be constant. Because of Eq. (4) the value of the temperature is given by Eq. (20). That is, the kinetic energy density for which the the entropy is maximum gives constant temperature.

\section{EXAMPLES AND SUMMARY}

Consider first the example of the linear harmonic oscillator. The potential is $V=\frac{1}{2} m \omega^{2} x^{2}$, where $m$ is the mass and $\omega$ is the frequency. The ground-state density is given by

$$
n(x)=\left(\frac{m \omega}{\pi \hbar}\right)^{1 / 2} e^{-m \omega x^{2} / \hbar} .
$$

As the ground-state kinetic energy is

$$
E_{k i n}=\frac{1}{4} \hbar \omega,
$$

the temperature is

$$
k T=\frac{1}{\beta}=\frac{\hbar \omega}{2} .
$$


Instead of Eq. (8) we have

$$
t(x)=\frac{1}{2} k \operatorname{Tn}(x)=\frac{n(x)}{2 \beta}
$$

as the degrees of freedom is one in the present example.

As a second example consider the Hydrogen atom in its ground state. The density is given by

$$
n(r)=\frac{1}{\pi}\left(\frac{Z}{a_{0}}\right)^{3} e^{-2 Z r / a_{0}}
$$

where

$$
a_{0}=\frac{\hbar^{2}}{m e^{2}}
$$

and $Z, r, m$ and $e$ are the atomic number, the radial distance, the electron mass and the magnitude of the electronic charge, respectively. As the ground-state kinetic energy is

$$
E_{k i n}=\frac{\hbar^{2} Z^{2}}{2 m a_{0}^{2}}
$$

the temperature is

$$
k T=\frac{1}{\beta}=\frac{Z^{2} m e^{4}}{3 \hbar^{2}} .
$$

In summary, we reopened the problem of thermodynamical view of the density functional theory. The theory is not unique because of the ambiguity of the kinetic energy density. Usually the everywhere positive gradient form is applied. Here we proved that it is possible to choose the kinetic energy density so that the local temperature be a constant for the whole system under consideration. Then, the kinetic energy density is proportional to the electron density and the temperature is proportional to the kinetic energy. One can select the kinetic energy density that maximizes the entropy. It was proved that this maximizing kinetic energy density corresponds to the constant temperature.

[1] S.K. Ghosh, M. Berkowitz and R.G. Parr, Proc. Natl. Acad. Sci. USA 81 (1984) 8028.

[2] S. K. Ghosh and R. G. Parr, Phys. Rev. A 34 (1986) 785.

[3] S.K. Ghosh and M. Berkowitz, J. Chem. Phys. 83 (1985) 2979. 
[4] R. G. Parr, K. Rupnik and S. K. Ghosh, Phys. Rev. Lett. 56, 1555(1986).

[5] C. Lee and R. G. Parr, Phys. Rev. A 35, 2377(1987).

[6] S. R. Gadre and R. D. Bendale, Int. J. Quant. Chem. 28, 311 (1985).

[7] S. R. Gadre, S. B. Sears, S. J. Chakravorty and R. D. Bendale, Phys. Rev. A 32, 2602(1985).

[8] S. R. Gadre, Phys. Rev. A 30, 620(1984).

[9] S. R. Gadre, S.A. Kulkani and I. H. Shrivastava, Chem. Phys. Lett.166, 445 (1990).

[10] S. R. Gadre, R. D. Bendale, S. P. Gejii, Chem. Phys. Lett.117, 138 (1985).

[11] Á. Nagy and R. G. Parr, Proc. of Ind. Acad. Sci. (Chem. Sci) 106 (1994) 217.

[12] Á. Nagy and R. G. Parr, J. Mol. Struct. (Theochem) 501 (2000) 101.

[13] Á. Nagy and R. G. Parr, Int. J. Quantum Chem. 58 (1996) 323.

[14] Á. Nagy, Proc. of Ind. Acad. Sci. (Chem. Sci) 106 (1994) 251.

[15] Á. Nagy, in Reviews of Modern Quantum Chemistry, ed. K. D. Sen (World Scientific, 2002) Vol.I, p.413.

[16] Á. Nagy, J. Mol. Struct Theochem 943 (2010) 48-52.

[17] P. Hohenberg and W. Kohn, Phys. Rev. 136 (1964) B864.

[18] W. Kohn and L. J. Sham, Phys. Rev. 140(1965) A1133.

[19] L. Cohen, J. Chem. Phys. 80 (1984) 4277. 